\title{
Variation in Performance of Platinum Electrodes with Size and Surface Roughness
}

\author{
Rylie A. Green*, Hamza Toor, Christopher Dodds and Nigel H. Lovell \\ Graduate School of Biomedical Engineering, University of New South Wales, \\ Sydney, 2052, Australia
}

(Received December 9, 2011; accepted January 23, 2012)

Key words: microelectrodes, platinum, injection limit, capacitance

Platinum (Pt) has been extensively used in medical electrodes and is proposed as a candidate for stimulation sites in retinal neuroprostheses due to its relative inertness and stability in biological environments. However, as implantable therapeutic devices are created with increasingly smaller dimensions, it is necessary to ensure that electrode properties are optimised. In this study, we present the variation in Pt electrode performance, which occurs as a result of reducing the electrode size. Additionally, the feasibility of laser roughening these electrodes is considered with respect to stability under chronic stimulation. It is shown that as the electrode diameter is decreased, the charge storage capacity (CSC) per unit area is increased twofold. Additionally, the frequency-dependent impedance per unit area decreases, resulting in an increase in the charge injection limit of up to 3.4 times in the biological environment. Finally, the stability of laser-roughened electrodes is demonstrated by continuous biphasic stimulation for more than 1 billion pulses at levels consistent with the activation of the neural retina.

\section{Introduction}

Microelectrodes are an integral component of many micro-electromechanical system (MEMS) devices, and with the advent of more sophisticated electronics and fabrication technologies, the manufacture of smaller electrodes has become achievable. Advances in photolithographic techniques, ${ }^{(1,2)}$ micromachining, ${ }^{(3)}$ laser patterning ${ }^{(4)}$ and ink-jet printing $^{(5)}$ have produced electrodes on the nanometer scale. ${ }^{(2)}$ These electrodes are typically fabricated from metals such as platinum (Pt), gold and $\mathrm{Pt}$ alloys, which have shown stability in implant devices such as the artificial retina, cochlear implant, and brain-machine interfaces. ${ }^{(1,4-6)}$ While all electrode systems proposed in the literature

*Corresponding author: e-mail: r.green@unsw.edu.au 
for specific devices are well characterised for electrical performance, there is only minimal data describing how these electrode properties change as they are fabricated with increasingly small surface areas. Cogan has observed that geometry and area affect the charge transfer properties of electrodes due to the nonuniformity in the current distribution that localizes the faradaic reactions to the perimeter or tip of an electrode. ${ }^{(7)}$

It has been found that all metallic electrodes, particularly Pt electrodes, have limited charge injection capability as a result of the water window constraints. ${ }^{(7)}$ Essentially, the water window describes the maximum voltage that can be applied to a single electrode interface, which when exceeded will lead to the irreversible electrolysis of water. The resulting electrolysis or metallic dissolution will depend on whether the electrode was anodically or cathodically polarised, but the potential damage to the interfacing neural tissue at the electrode surface is well defined. ${ }^{(8)}$ Shannon has even empirically derived equations that allow current injection to be specified on the basis of the electrode geometry, surface area and estimated distance between the target tissue and the electrode. ${ }^{(8)}$

Previous studies have determined that the maximum charge injection limit of electrodes widely varies and is dependent on the electrode material. The charge injection limits for Pt and platinum iridium (PtIr) alloys are reported as $0.05-0.15 \mathrm{mC} / \mathrm{cm}^{2}$ by Rose and Robblee. ${ }^{(9)}$ While some higher limits were reported in earlier studies, ${ }^{(10)}$ these increases are considered to be due to the extension of the stimulation period to allow faradaic reactions to be utilised. (7) Coating technologies have been explored for extending the safe charge injection limit of metals, and these include the use of iridium oxide (IrOx) and conducting polymers. ${ }^{(11,12)}$ The charge injection limit of IrOx (activated IrOx, thermal IrOx and sputtered $\mathrm{IrOx}$ ) ranged from $1-5 \mathrm{mC} / \mathrm{cm}^{2}$, compared with that of the conducting polymer, poly(3,4-ethylene dioxythiophene) (PEDOT) having a safe injection limit of $2-15 \mathrm{mC} / \mathrm{cm}^{2}$. (7) Specifically, PEDOT networks have been found to be porous at the nano- and microscale, and this markedly increases the interfacial surface and hence, charge transfer area. ${ }^{(13)}$

In artificial retina devices, it has been shown by trials of epiretinal prostheses that patients with retinitis pigmentosa (RP) require a charge injection of $0.016-0.879 \mu \mathrm{C}$ per phase for a visual percept to be observed. ${ }^{(14-16)}$ This amount of charge corresponds to $0.008-0.448 \mathrm{mC} / \mathrm{cm}^{2}$ if stimulated with a larger 500- $\mu \mathrm{m}$-diameter electrode and $0.033-$ $1.79 \mathrm{mC} / \mathrm{cm}^{2}$ if stimulated with a smaller $250-\mu \mathrm{m}$-diameter electrode. While the lower range of required stimulation is within the commonly reported Pt injection limits, these values were observed to be specific for patients who had retained light perception prior to the study. ${ }^{(15)}$ For older patients with no light perception prior to implantation, the perception threshold was at the higher end of the given range, ${ }^{(15)}$ and hence, beyond the commonly reported injection limit for Pt. Additionally, stimulation of vision using a more distant placement of devices, such as suprachoroidal and sclera placements, have been shown to require a higher charge injection per phase in animal models. ${ }^{(12,17)}$ It is clear that sufficient stimulation for useful vision across a wide range of patients will require a careful balance between electrode size and the charge injection capacity of the chosen material. While coating technologies show considerable promise in improving safe charge injection, PEDOT is not yet approved by regulatory bodies, and activated 
IrOx for vision prostheses is still under efficacy testing. ${ }^{(18)}$ As a result, these materials are unlikely to play a significant role in first-generation commercially viable artificial retinas.

A further confounding factor is the biological environment. Most of the literature on charge injection report values that are obtained in saline and saline variants such as phosphate buffers. However, protein adsorption is recognised as the first event following material-biofluid contact followed by cellular interactions that are mediated by these adsorbed proteins. ${ }^{(19)}$ Biomaterial interactions are dependent on surface topography, chemistry and mechanical properties of the micro- and nanoenvironments. There is little understanding of the effect these protein interactions have on electrode performance, other than observations of increased impedance, which would risk a further reduction in safe charge injection.

The consequence is that devices based on micro- (sub $100 \mu \mathrm{m}$ ) and nano- (sub 100 $\mathrm{nm}$ ) sized electrodes may not be able to safely supply sufficient charge for chronic stimulation applications. It has been proposed that patterning or roughening of the electrode surface may result in a sufficient increase in surface area to permit safe charge injection. ${ }^{(20)}$ In particular, laser roughening has been proposed as a method of increasing the charge transfer surface area of Pt microelectrodes while maintaining comparable biological performance, which does not significantly alter the implant biomaterial properties. ${ }^{(20,21)}$ It has been suggested that this technique can increase the surface area by up to five times, which potentially could increase the charge injection limit proportionally. ${ }^{(20)}$ However, the injection limit of these electrodes is not reported or systematically studied as a function of geometric electrode size. In this study, we aim to provide a systematic analysis of the Pt electrode size and laser roughening across a range of electrical performance metrics including frequency-dependent impedance, charge storage capacity and charge injection limit. Additionally, the effect of the biological environment is explored by performing analysis in protein-rich solutions as a model of the in vivo environment.

\section{Materials and Methods}

\subsection{Sample preparation}

The microelectrode arrays used in this project have been described. ${ }^{(22,23)}$ The materials used consisted of $99.95 \%$ pure Pt foil (Surepure Chemetals, USA) and poly(dimethyl siloxane) (PDMS) (MED-1000, NuSil, Carpinteria, CA, USA) mixed in a one-to-one ratio with hydrocarbon solvent ( $n$-heptane, Ajax Chemicals Ltd, Sydney, NSW, Australia). The electrode fabrication technique employed has been described by Dodds et al. (24)

Briefly, a base layer of $n$-heptane-diluted PDMS (1:1 ratio) was spin coated onto a standard microscope slide as a support substrate. A thickness of $60 \mu \mathrm{m}$ was achieved using 2,000 rpm for $90 \mathrm{~s}$ at room temperature $\left(21^{\circ} \mathrm{C}\right)$. The layer was cured at $60^{\circ} \mathrm{C}$ for 15 min. $25-\mu \mathrm{m}$-thick Pt was applied to the slide and the electrode array configuration was patterned onto the Pt foil using a numerically controlled laser (Nd:YAG, Firescan DPL Genesis Marker, CAB GmbH, Karlsruhe, Germany). Waste materials were 
removed manually and a second layer of PDMS/ $n$-heptane was applied using identical process parameters to fully insulate the Pt. Finally, the contacts/connection points of the electrodes were opened by a two-step process of first laser crystallising and then ablating the overlying PDMS. Samples were made with three electrode sizes by varying the diameter to either 250,500 , or $1,000 \mu \mathrm{m}$. Surface roughening is a by-product of the process and, as such, laser remelting of the surface was required to produce smooth samples for comparison at a diameter of $250 \mu \mathrm{m}$.

Optical profilometry was used to confirm the electrode surface area and roughness parameters. A GTK1-M Contour light interferometry profilometer (NewSpec, Australia) was used with a $20 \times$ objective in VSI mode to obtain a 3D rendering of the electrode surfaces. Vision 64 software, which is part of the GTH1-M system, was used to generate surface areas and roughness parameters. The particular parameters of interest were considered to be the real surface area and surface index (SI) and the roughness parameters $S_{\mathrm{q}}$ and $S_{\mathrm{z}} . S_{\mathrm{q}}$ is the root mean square (RMS) roughness that describes the finish of the electrode surface. It represents the deviation of the profile heights. $S_{z}$ is the average maximum profile of the peak-to-trough separations in the evaluation area. It is useful for evaluating the equivalence of the surface texture of the various electrodes where the presence of high peaks and deep troughs will be of functional significance.

Scanning electron microscopy (SEM) images were also generated to allow the visualisation of the conductive area of the electrode and provide qualitative observations of the electrode surface. Electrode arrays were placed in a JEOL Neoscope benchtop SEM (Coherent Scientific, Australia) and images were taken at $10 \mathrm{kV}$ with $500 \times$ magnification.

\subsection{Charge storage capacity}

Charge storage capacity (CSC) measurements were made by cyclic voltammetry (CV). The CV was performed using an eDaq potentiostat and eCorder unit (eDaq, Australia). Electrodes were prepared for CV by immersion in the electrolyte, phosphatebuffered saline (PBS, pH 7.58, Sigma Aldrich), and then excess oxygen was removed by bubbling nitrogen through the solution for $20 \mathrm{~min}$. An initial set of electrodes were used to establish the water window. A scan rate of $250 \mathrm{mV} / \mathrm{s}$ was used to sweep the potential at the working electrode between -1.5 and $1.5 \mathrm{~V}$, versus an isolated silver/silver-chloride $(\mathrm{Ag} / \mathrm{AgCl})$ reference electrode. This was performed in the PBS and used to identify the peaks consistent with irreversible reactions at the water window.

Subsequent cycling was performed within the water window from -0.6 to 0.8 $\mathrm{V}$ for up to 100 cycles on each electrode. The area within the curve was calculated using eChem software to obtain the CSC. Both the total CSC and cathodal CSC were calculated from twelve electrodes with mean values and standard deviation represented.

\subsection{Frequency-dependent impedance}

An InPhaze electrochemical impedance analyzer (InPhaze, Australia) was used to apply a sinusoidal potential waveform with $30 \mathrm{mV}$ (peak-to-peak or rms) to the working electrode submerged in PBS, controlled using an isolated $\mathrm{Ag} / \mathrm{AgCl}$ reference and a $\mathrm{Pt}$ counter electrode. Data points were collected from $1 \mathrm{mHz}-1 \mathrm{MHz}$, and impedance magnitude and phase were analysed. 


\subsection{Injection limits}

The potential transient response was measured in a three-electrode system with an isolated $\mathrm{Ag} / \mathrm{AgCl}$ reference electrode, low impedance Pt counter electrode and the test electrode immersed in either PBS or $15 \%$ horse serum (HS) loaded in RPMI cell culture medium (Sigma-Aldrich, Australia). Current controlled biphasic waveforms were applied to determine the charge injection limit. The maximum negative potential excursion $\left(E_{\mathrm{m}}\right)$ in the voltage transient, as indicated by the arrow in Fig. 1, is used to determine this limit. $E_{\mathrm{m}}$ was calculated by subtracting the access voltage $\left(V_{\mathrm{a}}\right)$, associated with the ohmic resistance of the electrolyte. $E_{\mathrm{m}}$ was measured to include the interpulse potential (IPP) following the delivery of the first phase of the stimulus. An interpulse delay of $0.01 \mathrm{~ms}$ was applied to increase the accuracy of the measurement. The charge injection limit was defined as the quantity of the charge that polarizes the electrode interface to the potential for water reduction $\left(E_{\mathrm{m}}=-0.6 \mathrm{~V}\right)$. The pulses were applied cathodic first, as is common practice for implant stimulations. The phase length was varied from $0.1-0.8 \mathrm{~ms}$ incrementally by $0.1 \mathrm{~ms}$ and the current was increased to obtain the limit. It is important to note that the stimulator used was developed for an implantable visual prosthesis and applies electrode shorting between pulses to recover the residual charge in the case of any charge imbalance.

For the measurement of charge injection limits in a biologically relevant electrolyte, $15 \%$ serum medium was placed on the electrode for $2 \mathrm{~h}$, facilitating protein interaction with the surface prior to measurement.

\subsection{Stability}

The stability of roughened electrodes was analysed in comparison with the smooth electrodes of $250 \mu \mathrm{m}$ diameter. Biphasic pulses were applied in 15\% HS-supplemented cell culture medium (RPMI) to determine the stability of laser-roughened surfaces when stimulated in an in vivo environment analogue. Stimulations were applied at $250 \mu \mathrm{A}$

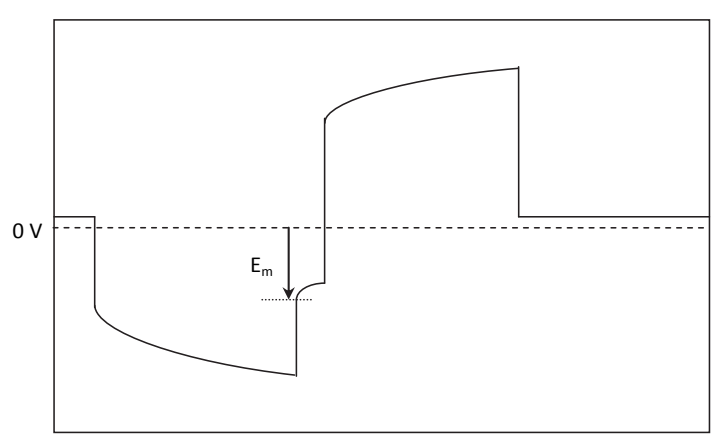

Fig. 1. Voltage transient on metallic electrode under charge-balanced biphasic stimulation measured with reference to $\mathrm{Ag} / \mathrm{AgCl}$ electrode. The arrow indicates the region that is limited to -600 $\mathrm{mV}$ according to the water window that is used to determine the charge injection limit. 
with $200 \mu$ s phase widths, which correlated with the charge injection threshold required to measure an evoked response in the visual cortex in previous in vivo experiments..$^{(11,17)}$ Electrodes were placed in an incubator at $37^{\circ} \mathrm{C}$ and $100 \%$ humidity, and the maximum voltage excursion across two electrode interfaces (bipolar stimulation) was measured daily for 50 days. Stimulation was constant at $250 \mathrm{~Hz}$ and the medium was replaced every 2 days to preserve the activity of the proteins.

\section{Results}

Electrodes were fabricated at three diameters and the smallest electrode was produced with both rough and smooth surface textures. The surface was characterised by SEM and optical profilometry to obtain the geometric and real surface areas for roughened electrodes (see Table 1). Additionally, the relevant surface roughness parameters are presented in Fig. 2, to establish that roughening via laser melt processes produced a

Table 1

Profilometry of electrodes at various diameters with a smooth vs rough comparison for $250 \mu \mathrm{m}(n=6)$.

\begin{tabular}{|c|c|c|c|c|}
\hline Diameter $(\mu \mathrm{m})$ & & & 500 & 1,000 \\
\hline Surface treatment & Smooth & Rough & Rough & Rough \\
\hline Nominal geometric area $\left(\mathrm{mm}^{2}\right)$ & 0.049 & 0.049 & 0.196 & 0.785 \\
\hline Manufactured geometric area $\left(\mathrm{mm}^{2}\right)$ & $0.041 \pm 0.006$ & $0.040 \pm 0.002$ & $0.205 \pm 0.039$ & $0.728 \pm 0.058$ \\
\hline Real area $\left(\mathrm{mm}^{2}\right)$ & $0.053 \pm 0.010$ & $0.104 \pm 0.009$ & $0.570 \pm 0.094$ & $1.826 \pm 0.119$ \\
\hline Surface index & $1.228 \pm 0.151$ & $2.622 \pm 0.189$ & $2.791 \pm 0.118$ & $2.515 \pm 0.169$ \\
\hline
\end{tabular}

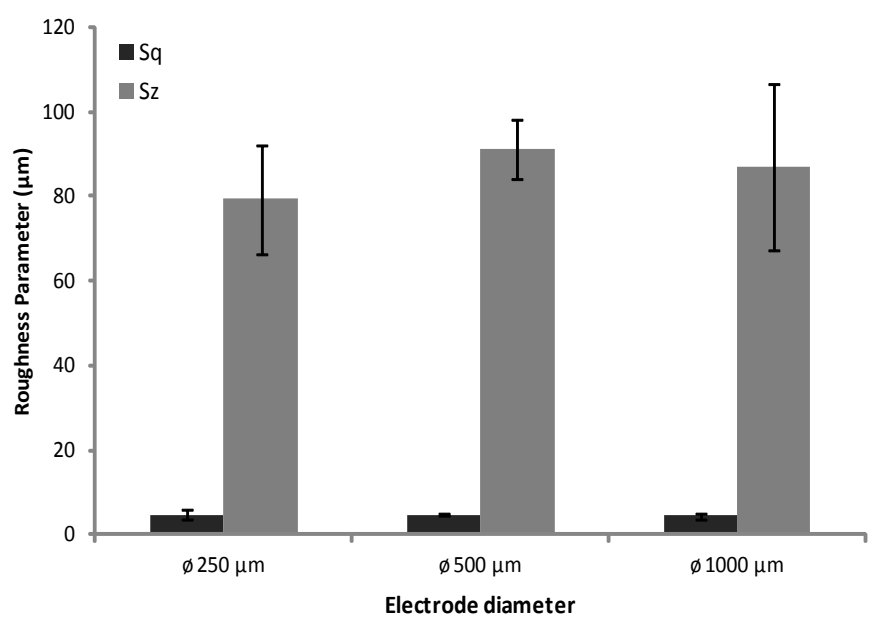

Fig. 2. Roughness parameters $S_{\mathrm{q}}$ and $S_{\mathrm{z}}$ for laser-roughened electrodes demonstrate that despite geometric size differences, the surface property is constant through equivalence in both peak-totrough height and deviation in the average profile. 
comparable surface for all electrode sizes. A representative image of the laser-roughened surface compared with the smooth surface is presented in Fig. 3.

The CSC values as determined by integrating the CV curve are presented in Table 2. These results show an increase in charge transfer occurring per unit area of the smaller roughened electrodes. It is observed from the CV plots shown in Fig. 4 that as the electrode diameter is decreased, the capacitive behaviour of the electrode is preserved in the bulk hysteresis curve. When normalised to the geometric area of the electrode, this translates to a higher CSC for the smaller electrodes.

Impedance measurements demonstrate that, as expected, the impedance of the electrodes increases as the size of the electrode decreases. However, in Fig. 5, when the impedance is normalised per unit area, the smaller $250-\mu \mathrm{m}$-diameter roughened electrodes have a lower impedance per unit area in the low-frequency range. At frequencies between 1 and $1,000 \mathrm{~Hz}$, the smallest electrodes also show lower phase angles, which correspond to a higher capacitance.

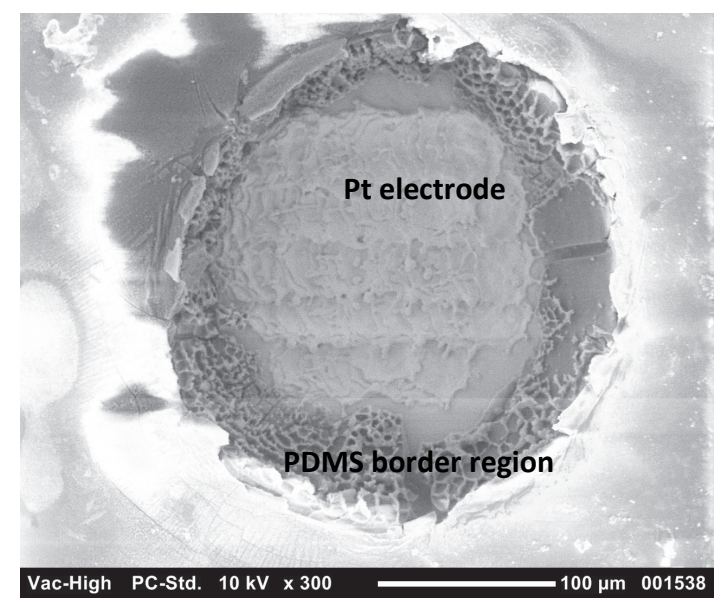

(a)

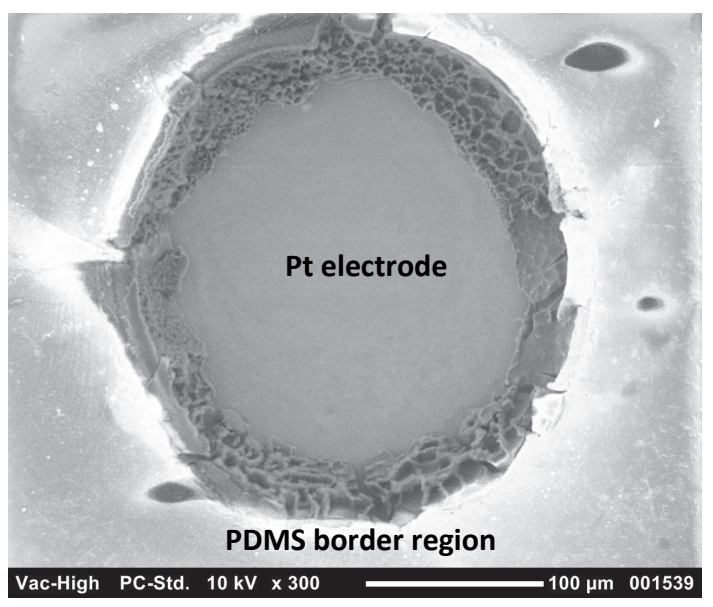

(b)

Fig. 3. SEM image of laser-roughened electrode (a) compared with a laser-smoothened electrode (b) with nominal $250 \mu \mathrm{m}$ diameter.

Table 2

Charge storage capacity of Pt electrodes normalized to geometric area $(n=6)$.

\begin{tabular}{lrrrr}
\hline Diameter $(\mu \mathrm{m})$ & \multicolumn{2}{c}{250} & 500 & $\begin{array}{c}1,000 \\
\text { Surface treatment }\end{array}$ \\
\cline { 2 - 3 } & Smooth & Rough & Rough & Rough \\
\hline $\mathrm{CSC}\left(\mathrm{mC} / \mathrm{cm}^{2}\right)$ & $4.1 \pm 0.4$ & $10.4 \pm 1.2$ & $4.3 \pm 0.8$ & $4.4 \pm 0.3$ \\
Cathodic $\mathrm{CSC}\left(\mathrm{mC} / \mathrm{cm}^{2}\right)$ & $2.4 \pm 0.4$ & $6.1 \pm 1.4$ & $2.2 \pm 0.5$ & $2.1 \pm 0.1$ \\
\hline
\end{tabular}




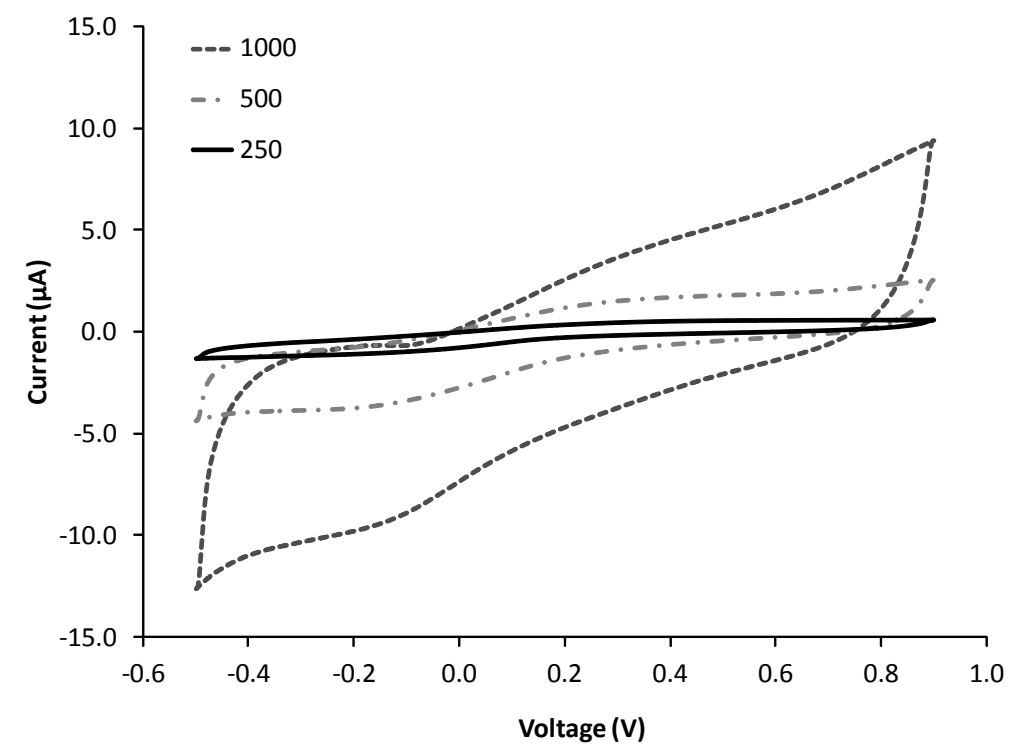

(a)

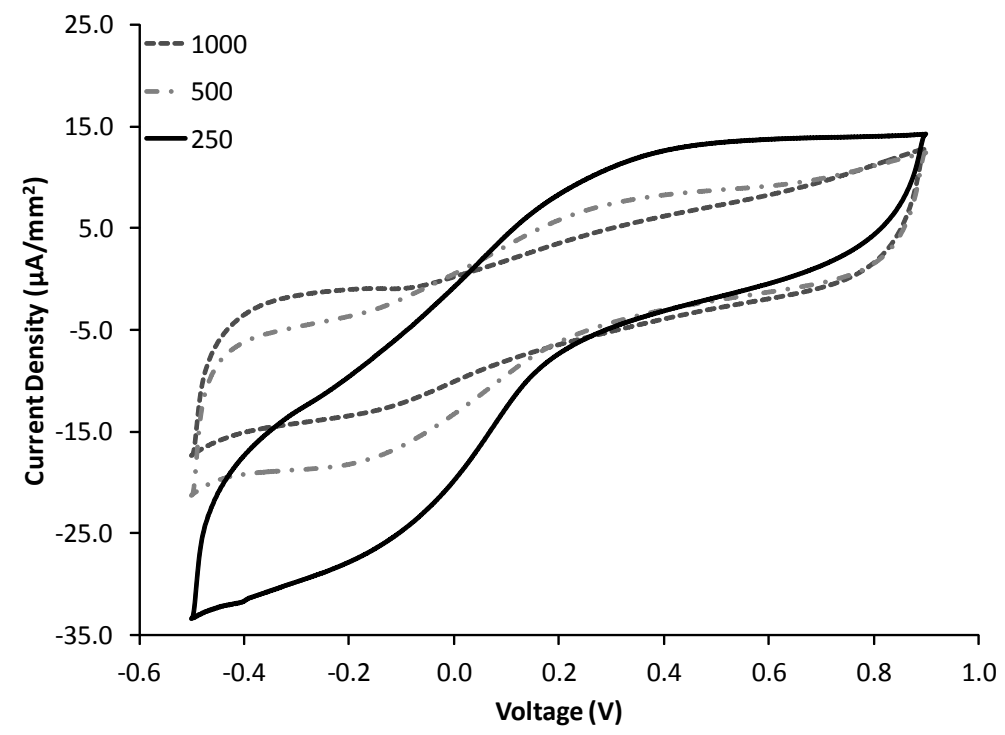

(b)

Fig. 4. Sample CV curves of roughened microelectrodes of various sizes analysed in PBS vs Ag/ $\mathrm{AgCl}$ reference and large Pt counter. The raw data (a) is compared to data normalised to current density (b). 


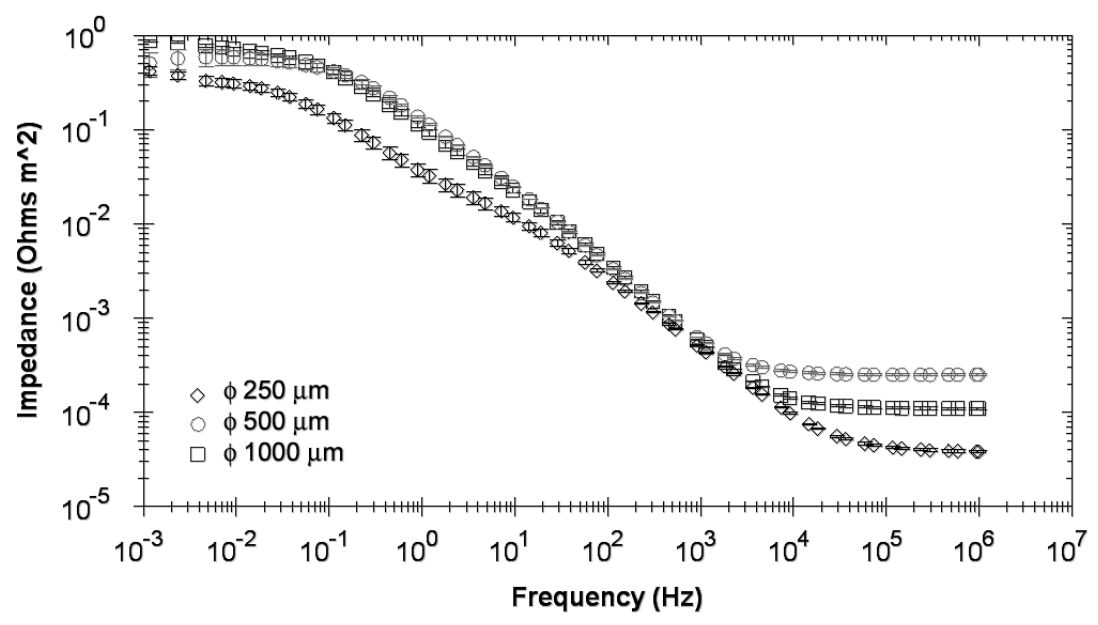

(a)

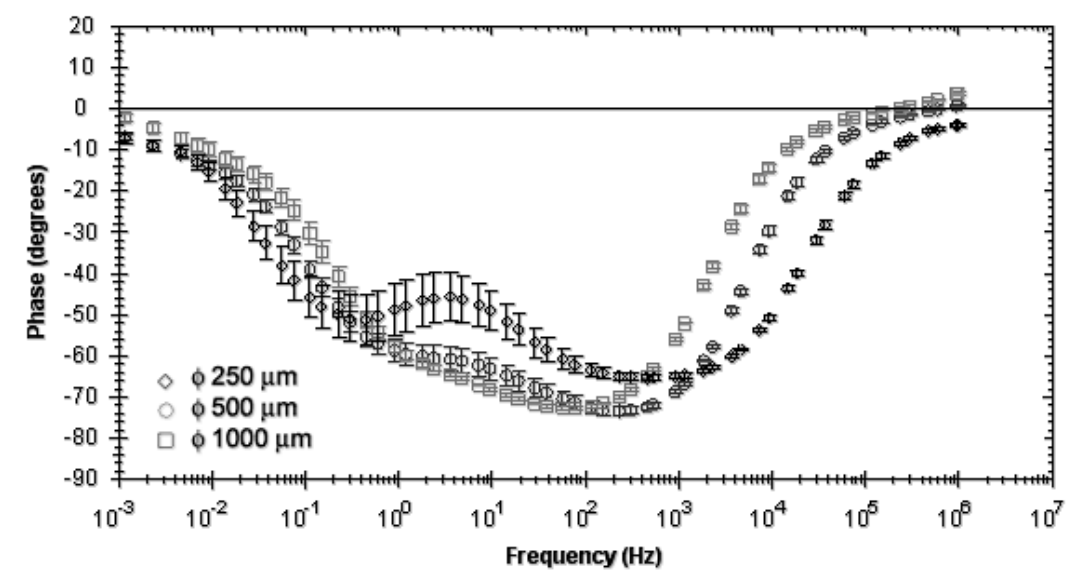

(b)

Fig. 5. Frequency-dependent impedance magnitude (a) and phase lag (b) of electrodes of varying sizes, analysed in PBS with $30 \mathrm{mV}$ sinusoidal pulses $(n=4)$.

The charge injection limit was analysed for all sizes and surface topographies in both $0.9 \%$ saline and high-serum cell culture medium, resulting in the curves shown in Figs. 6 and 7. The 500- $\mu \mathrm{m}$-diameter electrodes were found to be almost identical to the $1,000-\mu \mathrm{m}$-diameter electrodes in saline, but the $250-\mu \mathrm{m}$-diameter electrodes were found to have an injection limit that ranged from 2.8 to 3.7 times higher than that of the larger 


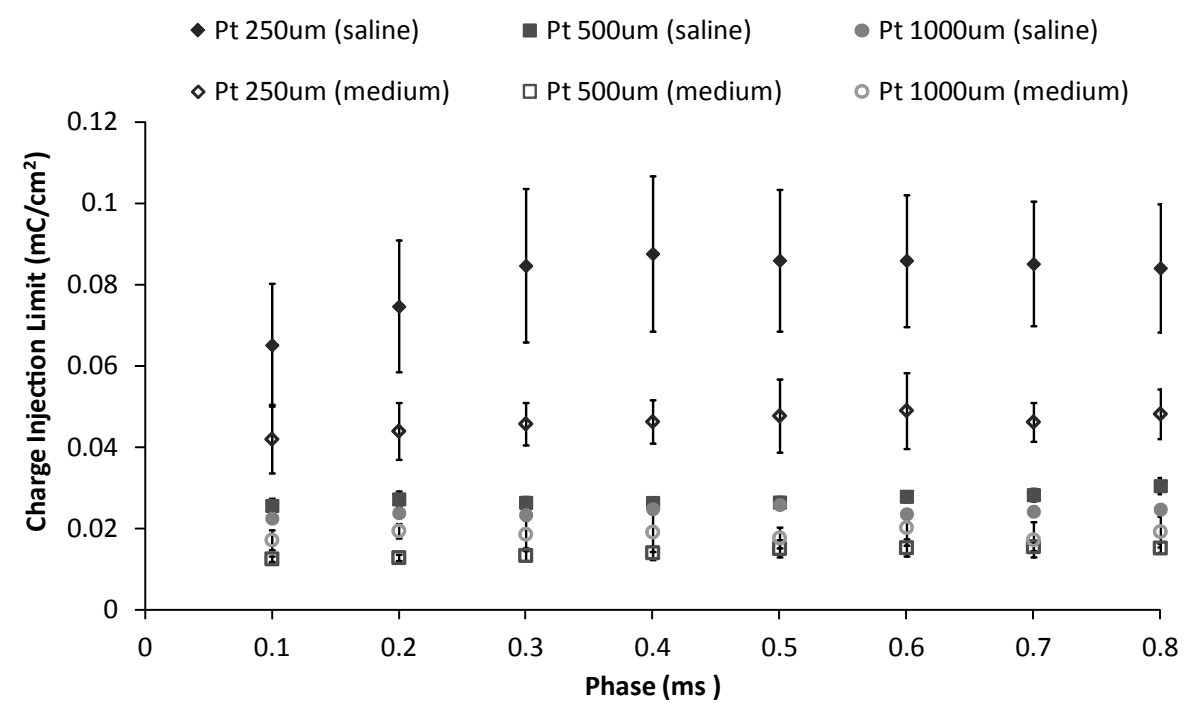

Fig. 6. Charge injection variability with roughened electrodes of different sizes in both saline and medium $(n=4)$.

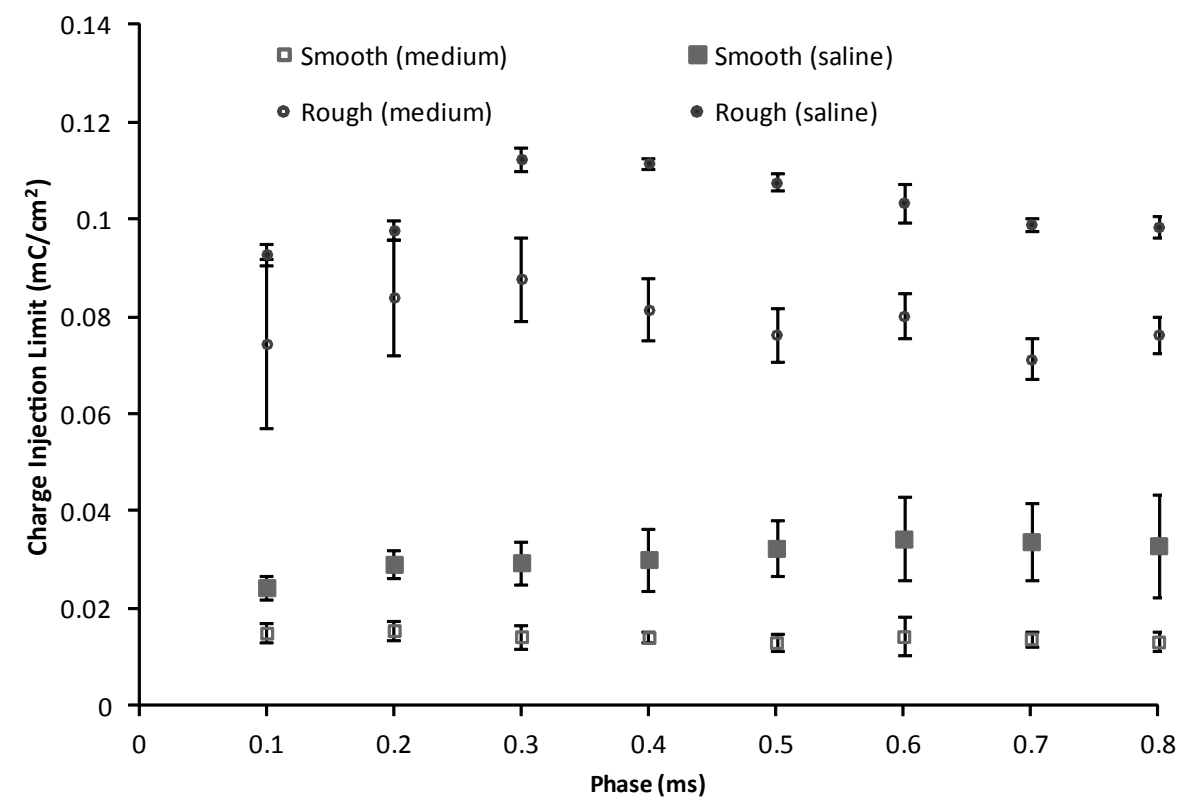

Fig. 7. Comparison of charge injection limit for smooth versus rough electrodes of $\phi 250 \mu \mathrm{m}$ in $0.9 \%$ saline and $15 \%$ serum-loaded medium $(n=28)$. 
electrodes. When tested in the serum-loaded medium, all the charge injection limits were observed to decrease. This reduced the charge injection limit benefit provided by small electrodes to 2.2 to 2.7 times in comparison with that of the largest $1,000-\mu \mathrm{m}$-diameter electrodes. The smallest electrodes still afforded up to 3.4 times more charge injection than the intermediate $500-\mu \mathrm{m}$-diameter electrodes.

Figure 7 depicts the variation of charge injection limit when the surface topography is altered to present a smoother interface. A significant concern previously encountered for roughened electrodes is the potential for protein blocking; however, it can be seen that the effect of the high-serum medium on the charge injection limit is minimal compared with the significant increase in the injection limit offered by roughening.

Long-term stimulation studies have been conducted on both the roughened and smooth electrodes with a $250 \mu \mathrm{m}$ diameter. Stimulations were applied using parameters that had previously been shown to elicit a response in the visual cortex of feline models (biphasic current amplitude of $250 \mu \mathrm{A}$ and phase length of $0.2 \mathrm{~ms}$ ). Both electrode types demonstrated stable end-of-phase impedance across a period of 1.3 billion cycles or 60 days. The testing was conducted in high-serum medium that was replaced every 48 $\mathrm{h}$ to ensure that functional proteins were present at the interface. The average voltage measured between roughened electrode pairs was $1.56 \pm 0.3 \mathrm{~V}$ compared with the smooth electrodes with an average voltage of $3.42 \pm 0.5 \mathrm{~V}$.

\section{Discussion}

Laser roughening of microelectrodes produces a surface that increases the available charge transfer area. Previous reports have considered the roughening of electrodes, but have not considered how roughening might affect planar electrodes of different sizes. In these studies, it has been determined that as the electrode size is decreased, there is an increase in the benefit of the laser roughening processes.

The laser roughening process presented is largely a melt process that was initially described by Schuettler et al. as a by-product of laser micromachining.(4) In this study, it was shown that despite this process being mediated by thermal effects, there was no significant difference in the roughness parameters and consequent surface area increase imparted to electrodes of different sizes. All the electrodes had a real surface area that was approximately 2.6 times larger than the geometric area. This degree of laser roughening has been shown to produce a surface that is both noncytotoxic and suitable for the attachment of cells. ${ }^{(21)}$

It is important to note that the electrodes used in this study are considered macroelectrodes. The planar electrode arrays used in vision prostheses are similar to those that form the stimulating array in cochlear implants. The size range has been chosen on the basis of the device requirement for larger electrodes of greater than 200 $\mu \mathrm{m}$ diameter to safely stimulate potentially distal neurons ${ }^{(25)}$ in comparison with cortical prostheses where microelectrodes usually penetrate into and are in contact with the target tissue. However, the visual field imparted by a vision prosthesis is directly related to the size of the stimulated area of the retina, and hence, the overall dimensions of the electrode array, ${ }^{(26)}$ which are determined from both the electrode diameter and packing 
limitations of the fabrication process. A study by Onnela et al. has suggested that either novel electrode materials or surface treatment methods will be required to meet the charge injection needs of a high resolution of vision prosthesis. ${ }^{(25)}$

In this study, it has been shown that the electrodes with a small area have a proportionally higher increase in charge transfer area both in the charge storage capacity when voltage is cycled and in the charge injection limit. It is proposed that this occurs through two mechanisms: (i) edge effects and (ii) a change in the shape of the electric field. Charge distribution on electrodes is known to be nonuniform and concentrated at the edges. ${ }^{(27)}$ When the edge area is increased relative to the electrode diameter, the higher charge-carrying capacity of the border region dominates the electrode performance. Additionally, it is proposed that the electric field shape can change as the electrode is downsized. The electric field of a larger electrode, in this case, 1,000 $\mu \mathrm{m}$ diameter, is dominated by linear diffusion processes of the ions. As the electrode size is reduced to $250 \mu \mathrm{m}$ diameter, the electrical field is proposed to take on a more hemispherical shape, increasing the charge accessibility to the electrode surface per unit area. This phenomenon was previously described by Cogan with respect to sputtered IrOx electrodes, ${ }^{(7)}$ which were systematically decreased in surface area from the macroto microscale.

This increase in the charge injection limit observed on smaller electrodes may provide one option for increasing the charge transfer capability of Pt electrodes without requiring the use of surface coatings. This increase has the potential to allow safe charge injection at biologically relevant levels such as those recommended for eliciting phosphenes in patients with retinal degeneration. With appropriate sizing, this could also provide sufficient charge injection to meet the needs of the more distal implants such as those with suprachoroidal and intrascleral placements, which have recently been proposed by several research groups developing vision prostheses. ${ }^{(28-30)}$

Despite increases in charge transfer capacity, fabrication challenges may limit the realisation of the benefit of smaller electrodes. As shown in Fig. 8, to improve upon the maximum charge/phase performance of a 1,000- $\mu \mathrm{m}$-diameter electrode, at least 11

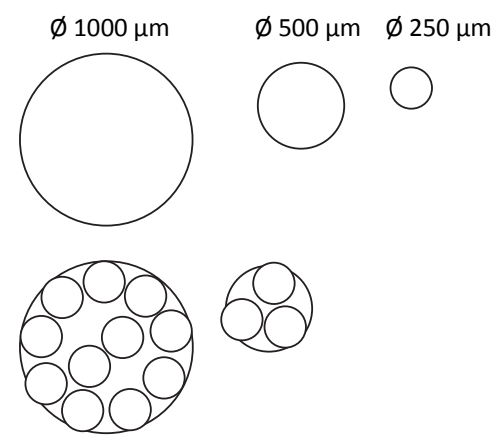

Fig. 8. Scaled electrode size comparison for increased charge injection performance. 
electrodes with the smallest $250 \mu \mathrm{m}$ diameter would be required within the same space. Similarly, 3 of the $250-\mu \mathrm{m}$-diameter electrodes would be required to replace a $500-\mu \mathrm{m}-$ diameter electrode. Many researchers suggest that with greater numbers of electrodes, the resolution of perceived vision would be improved. ${ }^{(31-33)}$ Additionally, there have been sufficient advances in fabrication technologies that allow these higher density arrays of smaller electrodes to be individually addressable. However, it must be recognised that some clinical trials have shown a negligible grading in the visual response with the downsizing of electrodes. ${ }^{(15)}$ A possible alternative approach may involve either the use of multiple smaller electrodes stimulated in parallel or "ganged" to achieve a higher total charge or the generation of multiple smaller electrode openings overlying a larger electrode to give a perforated appearance. The question, however, remains as to whether these approaches would retain the hemispherical charge access or result in a linear distribution.

In this study, the charge injection limit for roughened electrodes is only comparable to values reported by Rose and Robblee, ${ }^{(9)}$ and the smoothened electrodes have an even lower injection limit. This is thought to be related to the chemical changes that occur at the interface during laser roughening where melt effects are observed. In previous studies, it was shown that oxides of Pt were formed during this process. ${ }^{(21)}$ Specifically, in XPS analyses, the Pt $4 \mathrm{f7}$ peak shifted to a higher energy level, and increases in oxygen spectra were observed at binding energies consistent with the formation of both $\operatorname{Pt}(\mathrm{OH})_{4}$ and $\mathrm{PtO}_{2} \cdot{ }^{(34)}$ While oxides generally are known to increase CSC by engaging reversible faradaic reactions within the water window, and this is observed for these electrodes, they can reduce the charge injection by limiting the area of double-layer charging. It is expected that newer laser processing techniques using picosecond pulsing, which do not involve bulk melting of the surface, can achieve a more controlled electrode patterning without imparting chemical modifications.

Protein interactions as determined by media injection limits showed a reduced limit for both rough and smooth electrodes. The effect was more pronounced for smaller electrodes, possibly due to increased roughness of the insulating material at the border region. Roughened polymers and metals are known to provide improved surface properties for cell attachment, and this is considered to be the result of better serum protein binding. ${ }^{.35,36)}$ There were some concerns that this increase in protein interaction would reduce the benefit of roughening in comparison with smoother electrodes. Figure 7 demonstrates that despite an average drop in the injection limit of $30 \%$, there was still a 2- to 3-fold increase in the limit provided by the roughened electrodes, which was more prominent at shorter phase lengths.

In the literature, electrochemical cycling has been used to roughen Pt electrodes. ${ }^{(37)}$ This mechanism of surface roughening involves oxide growth as a result of oxidation and reduction reactions. Oxygen ions penetrate the topmost layer of the $\mathrm{Pt}$, and when reduced, have to leave the metal lattice, resulting in surface roughening through lattice restructuring and material loss. ${ }^{(37)}$ One of the aspects to this study was to confirm that laser roughening did not result in an undesirable surface, which would affect the stability of these electrodes under typical biphasic stimuli. It was considered that if voltages occurred on the roughened electrode surface, which breached oxidation 
and/or reduction potentials, oxides in the $\mathrm{Pt}^{(21)}$ would potentially change the surface structure and ultimately result in an unstable interface with material loss. The peaks of roughened Pt may experience localised increases in charge density, which could result in Pt restructuring or dissolution. Long-term stimulation was performed in high serum medium, accruing more than 1 billion pulses without experiencing a significant change in stability. It is proposed that the adsorbed protein, which reduced the charge injection limit through protein blocking, also reduces Pt dissolution. ${ }^{(7)}$

Electrode size has been shown to have a significant impact on charge transfer properties. Despite findings that the electrode size does not affect threshold values in epiretinal prostheses, ${ }^{(15)}$ this effect has not been systematically investigated with respect to alternative device placements such as suprachoroidal and scleral placements, in the clinical setting. Additionally, in vitro studies by Jensen et al. ${ }^{(38)}$ showed that smaller $125-\mu \mathrm{m}$-diameter electrodes had lower thresholds for retinal ganglion cell activation than the 500- $\mu \mathrm{m}$-diameter electrodes used in the same study. These findings suggest that lower thresholds may be used to elicit vision percepts using smaller electrodes.

\section{Conclusions}

In summary, smaller electrodes that are laser roughened may provide superior charge transfer properties. In artificial retina where it has been proposed that electrode size may not be the determining factor in perceived vision, ganging of multiple smaller electrodes or fabricating smaller pseudoarrays over a larger electrode through a perforated approach may be an alternative option for creating the required electrode size with improved injection properties. To elicit the benefits of smaller electrodes, it is expected that dense packing will be required, which will also provide flexibility for high-resolution vision prostheses in future devices. There are also significant benefits of roughening these electrodes to further improve the charge transfer properties. Future work will explore laser roughening processes that do not modify the bulk surface chemistry but rather pattern the electrode with controlled, uniform nodular structures.

\section{Acknowledgements}

The authors would like to thank Stuart Cogan from EIC Laboratories for his expert advice and review of data. Funding was provided in part through Bionic Vision Australia, a special initiative of the Australian Research Council.

\section{References}

1 E. Kim, J.-M. Seo, S. Woo, J. Zhou, H. Chung and S. Kim: Sensors 8 (2008) 5845.

2 P. Sun, Z. Zhang, J. Guo and Y. Shao: Anal. Chem. 73 (2001) 5346.

3 S. Rajaraman, J. A. Bragg, J. D. Ross and M. G. Allen: J. Micromech. Microeng. 21 (2011) 085014.

4 M. Schuettler, S. Stiess, B. King and G. J. Suaning: J. Neural Eng. 2 (2005) 121.

5 S. H. Ko, H. Pan, C. P. Grigoropoulos, C. K. Luscombe, J. M. J. Fréchet and D. Poulikakos: Nanotechnology 18 (2007) 345202. 
6 J. M. Seo, S. J. Kim, H. Chung, E. T. Kim, H. G. Yu and Y. S. Yu: Mater. Sci. Eng. C 24 (2004) 185.

7 S. F. Cogan: Ann. Rev. Biomed. Eng. 10 (2008) 275.

8 R. V. Shannon: IEEE Trans. Biomed. Eng. 39 (1992) 424.

9 T. L. Rose and L. S. Robblee: IEEE Trans. Biomed. Eng. 37 (1990) 1118.

10 S. B. Brummer and M. J. Turner: IEEE Trans. Biomed. Eng. 24 (1977) 59.

11 S. Venkatraman, J. Hendricks, Z. A. King, A. J. Sereno, S. Richardson-Burns, D. Martin and J. M. Carmena: IEEE Trans. Neural Syst. Rehabil. Eng. 19 (2011) 307.

12 R. A. Green, F. Devillaine, C. Dodds, P. Matteucci, S. Chen, P. Byrnes-Preston, L. A. PooleWarren, N. H. Lovell and G. J. Suaning: Proc. 32nd Ann. Int. Conf. IEEE Engineering in Medicine and Biology Society (IEEE, NJ, USA, 2010) p. 6769.

13 X. T. Cui and D. D. Zhou: IEEE Trans. Neural Syst. Rehabil. Eng. 15 (2007) 502.

14 M. S. Humayun: Vision Res. 39 (1999) 2569.

15 C. de Balthasar, S. Patel, A. Roy, R. Freda, S. Greenwald, A. Horsager, M. Mahadevappa, D. Yanai, M. J. McMahon, M. S. Humayun, R. J. Greenberg, J. D. Weiland and I. Fine: Invest. Ophthalmol. Visual Sci. 49 (2008) 2303.

16 M. Mahadevappa, J. D. Weiland, D. Yanai, I. Fine, R. J. Greenberg and M. S. Humayun: IEEE Trans. Neural Syst. Rehabil. Eng. 13 (2005) 201.

17 Y. T. Wong, S. C. Chen, J. M. Seo, J. W. Morley, N. H. Lovell and G. J. Suaning: Vision Res. 49 (2009) 825.

18 G. Roessler, T. Laube, C. Brockmann, T. Kirschkamp, B. Mazinani, M. Goertz, C. Koch, I. Krisch, B. Sellhaus, H. K. Trieu, J. Weis, N. Bornfeld, H. Röthgen, A. Messner, W. Mokwa and P. Walter: Invest. Ophthalmol. Visual Sci. 50 (2009) 3003.

19 B. Kasemo and J. Gold: Adv. Dent. Res. 13 (1999) 8.

20 M. Schuettler: Proc. 29th Ann. Int. Conf. IEEE EMBS (IEEE, NJ, USA, 2007) p. 186.

21 R. A. Green, J. S. Ordonez, M. Schuettler, L. A. Poole-Warren, N. H. Lovell and G. J. Suaning: Biomaterials 31 (2010) 886.

22 M. Schuettler, S. Stiess, B. V. King and G. J. Suaning: J. Neural Eng. 2 (2005) 121.

23 G. J. Suaning, M. Schuettler, J. S. Ordonez and N. H. Lovell: Proc. 3rd Int. IEEE EMBS Conf. on Neural Engineering (IEEE, NJ, USA, 2007) pp. 5-8.

24 C. W. D. Dodds, Y. T. Wong, P. J. Byrnes-Preston, M. Rendl, N. H. Lovell and G. J. Suaning: Proc. 4th Int. IEEE/EMBS Conf. in Neural Engineering (IEEE, NJ, USA, 2009) p. 88.

25 N. Onnela, H. Takeshita, Y. Kaiho, T. Kojima, R. Kobayashi, T. Tanaka and J. Hyttinen: Biomed. Mater. Eng. 21 (2011) 83.

26 H. Ameri, T. Ratanapakorn, S. Ufer, H. Eckhardt, M. S. Humayun and J. D. Weiland: J. Neural Eng. 6 (2009) 035002.

27 J. T. Rubinstein, F. A. Spelman, M. Soma and M. F. Suesserman: IEEE Trans. Biomed. Eng. 34 (1987) 864.

28 Y. T. Wong, S. C. Chen, Y. A. Kerdraon, P. J. Allen, M. F. McCombe, J. W. Morley, N. H. Lovell and G. J. Suaning: Proc. 30th Ann. Int. Conf. IEEE (IEEE, NJ, USA, 2008) p. 1789.

29 J. A. Zhou, S. J. Woo, S. I. Park, E. T. Kim, J. M. Seo, H. Chung and S. J. Kim: J. Biomed. Biotechnol. 2008 (2008) 547.

30 K. Nakauchi, T. Fujikado, H. Kanda, T. Morimoto, J. S. Choi, Y. Ikuno, H. Sakaguchi, M. Kamei, M. Ohji, T. Yagi, S. Nishimura, H. Sawai, Y. Fukuda and Y. Tano: Graefe's Arch. Clin. Exp. Ophthalmol. 243 (2005) 169.

31 A. Hung, D. Zhou, R. Greenberg and J. W. Judy: Proc. 15th IEEE Int. Conf. on Micro Electro Mechanical Systems (IEEE, NJ, USA, 2002) p. 56.

32 R. A. Green, N. H. Lovell, G. G. Wallace and L. A. Poole-Warren: Biomaterials 29 (2008) 3393. 
33 D. C. Rodger, A. J. Fong, W. Li, H. Ameri, A. K. Ahuja, C. Gutierrez, I. Lavrov, H. Zhong, P. R. Menon, E. Meng, J. W. Burdick, R. R. Roy, V. R. Edgerton, J. D. Weiland, M. S. Humayun and Y.-C. Tai: Sens. Actuators, B 132 (2008) 449.

34 M. Peuckert, F. P. Coenen and H. P. Bonzel: Electrochim. Acta 29 (1984) 1305.

35 S. K. Nishimoto, M. Nishimoto, S. W. Park, K. M. Lee, H. S. Kim, J. T. Koh, J. L. Ong, Y. Liu and Y. Yang: Int. J. Oral Maxillofac. Implants 23 (2008) 675.

36 R. A. Green, G. J. Suaning, L. A. Poole-Warren and N. H. Lovell: Proc. 4th Int. IEEE/EMBS Conf. on Neural Engineering (NER) (IEEE, NJ, USA, 2009) p. 60.

37 A. Reiner, B. Steiger, G. G. Scherer and A. Wokaun: J. Power Sources 156 (2006) 28.

38 R. J. Jensen, O. R. Ziv and J. F. Rizzo: Invest. Ophthalmol. Visual Sci. 46 (2005) 1486. 